\title{
Optical and Microcantilever-Based Sensors for Real-Time In Situ Characterization of High-Level Waste (81924)
}

\section{Research Objective}

Fundamental research is being conducted to develop sensors for strontium that can be used in real-time to characterize high-level waste (HLW) process streams. Two fundamentally different approaches are being pursued, which have in common the dependence on highly selective molecular recognition agents. In one approach, an array of chemically selective sensors with sensitive fluorescent probes to signal the presence of the constituent of interest are coupled to fiber optics for remote analytical applications. The second approach employs sensitive microcantilever sensors that have been demonstrated to have unprecedented sensitivity in solution for $\mathrm{Cs}^{+}$and $\mathrm{CrO}_{4}{ }^{-}$. Selectivity in microcantilever-based sensors is achieved by modifying the surface of a gold-coated cantilever with a monolayer coating of an alkanethiol derivative of the molecular recognition agent. The approaches are complementary since fiber optic sensors can be deployed in the highly alkaline environment of HLW, but a method of immobilizing a fluorescent molecular recognition agents in a polymer film or bead on the surface of the optical fiber has yet to be demonstrated. The microcantilever-based sensors function by converting molecular complexation into surface stress, and they have been demonstrated to have the requisite sensitivity. However, we will investigate method of protecting $\mathrm{Si}$ or $\mathrm{SiN}$ microcantilever sensors in the highly alkaline environment of HLW while maintaining high selectivity. One objective of this project is to develop $\mathrm{Sr}(\mathrm{II})$ molecular recognition agents with rapidly established equilibria needed for real-time analysis, and initial research will focus on calixarene-crown ethers as a platform. Sensors for alkali metal ions, hydroxide, and temperature will be part of the array of sensor elements that will be demonstrated in this program for both the cantilever and fiber optic sensor approaches.

\section{Research Progress and Implications}

This report summarizes the research as of June, 2002, in the first year of a three year project. Specific goals in this program are to develop a selective complexation agent for $\mathrm{Sr}$ (II) based on calixarene crown ether chemistry, and then adapt this chemistry to fluorescence sensors and cantilever sensors. In addition, we are going to utilize previously developed $\mathrm{Cs}^{+}$selective 1,3-alt-calix[4]arene-benzocrown-6 compounds with a cyanoanthracene fluorophore group to develop fiber optic sensors that tolerates a high ionic background, highly alkaline environment while maintaining good sensitivity. Another goal in the initial stage of the project was to investigate passivating layers and attachment chemistry for microcantilever sensors that is tolerant of strongly alkaline solutions.

Arrays of sensors based on fiber optics are prepared by attaching the indicating chemistries to microspheres followed by randomly distributing the microspheres into the optical fiber arrays. Therefore, a robust method for encoding and registering the microspheres after assembly is required. The Tufts team has developed new methods for registering populations of microspheres in random arrays. A computer algorithm has been developed that enables the different microsphere types to be recognized and positionally registered. This system has been incorporated into our optical instrumentation to make the registration process seamless. The program is designed to ignore outliers in order to avoid making erroneous measurements. The next step in the research will be to attach the indicating chemistries developed at ORNL onto microspheres and to test the responses of the individual microspheres after immobilization. The 
goal is to develop a library of microspheres that will enable multiple analytes to be measured simultaneously in high level waste.

The synthesis of a calix[4]arene based $\mathrm{Sr}^{2+}$ probe for an optical sensor was carried out by alkylation of the lower rim of calix[4]arene twice as shown in the following scheme.
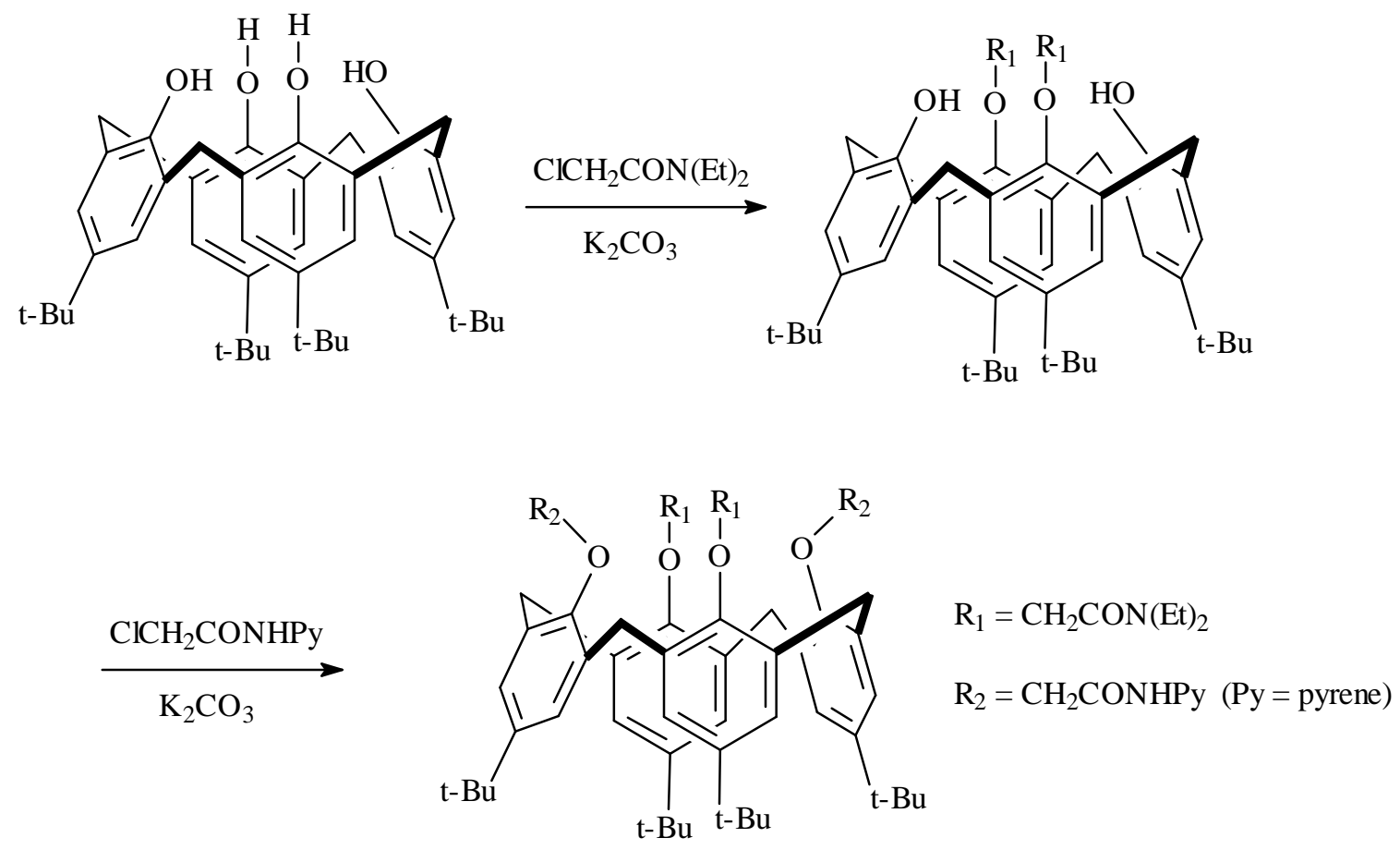

The first reaction adds an amide functionality that has been demonstrated to give $\mathrm{Sr}^{2+}$ selectivity to calixarene ligands in the cone conformation. The second reaction adds the indicating fluorescent pyrene group. Our preliminary results show that the fluorescence profile of this probe changes upon complexation with $\mathrm{Sr}^{2+}$. In the absence of $\mathrm{Sr}^{2+}$, both monomer (370nm) and

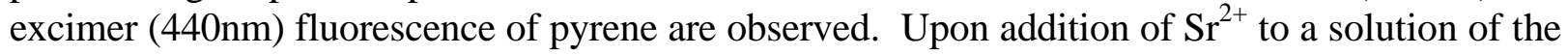
sensor molecule, the fluorescence of the excimer decreases while the monomer fluorescence peak increases. The selectivity of this probe is under investigating.

Investigations of $\mathrm{Cs}^{+}$selective calix[4]benzocrown-6 ligands containing a cyanoanthracene PET fluorophore continues with an emphasis on discovering the medium dependence of the emission enhancement following $\mathrm{Cs}^{+}$complexation. An experiment using Cs-137 radioactive tracer is in progress to separate the medium dependence of the ligand loading from the medium dependence of the PET effect.

An investigation of chemistry to passivate the surface of Si or SiN microcantilevers to the strongly alkaline medium of high level waste using siloxane and other metal oxide chemistry is in progress. 


\section{Planned Activities}

The objective of our investigations at the end of three years is to make arrays of cantilever sensors and fiber optic sensors for $\mathrm{Cs}^{+}, \mathrm{K}^{+}, \mathrm{Na}^{+}$, and $\mathrm{Sr}^{2+}$, based on known crown ether chemistry. We are planning to test these fiber optic and cantilever arrays first in cold waste simulant and then in actual Hanford tank waste. We will utilize literature formulations to add temperature and $\mathrm{OH}^{-}$sensor elements to the fiber optic and microcantilever arrays. The research in the next year will focus on improving stability in strong base solutions, and on gaining selectivity and sensitivity for the $\mathrm{Sr}^{2+}$ sensor elements.

\section{Information Access}

The following publication appeared in print during the year.

Hai-Feng Ji, Reza Dabestani, Gilbert M. Brown and Robert L. Hettich, "Synthesis and Sensing Behavior of Cyanoanthracene Modified 1,3-alternate Calix[4]benzocrown-6: a New Class of CsSelective Optical Sensors," J. Chem. Soc. Perkin Trans 2, 2001, 585-595. 